\title{
Estudio comparativo de la estadificación de pacientes con EPOC según GOLD 2007, 2011 y 2019
}

\author{
JOANNA SANTELIZ C.*
}

\section{Comparative study of the categorization of COPD patients based on GOLD 2007, 2011 and 2019}

Introduction: Chronic Obstructive Pulmonary Disease (COPD) affects 260 million people worldwide and it is thought to become the third leading cause of mortality by the year 2020. Materials and Methods: A transversal descriptive observational study was conducted to compare the categorization of a group of Venezuelan COPD patients according to the Global Initiative for Chronic Obstructive Lung Disease (GOLD) 2007, 2011 and 2019. Results: Eighty-nine (89) patients with a mean age of $66.7 \pm$ 0.9 years were included, $60.7 \%$ were male and $82 \%$ smokers. $14.6 \%$ of the patients had mild COPD, $36 \%$ moderate COPD, $41.6 \%$ severe COPD and $7.9 \%$ very severe COPD. Cohen's Kappa coefficient value between mMRC and COPD Assessment test (CAT) was 0,529 (GOLD 2011) and 0,555 (GOLD 2019). Conclusions: 1) the lack of concordance between FEV $V_{1}$ values, degree of dyspnea and history of exacerbations impacts COPD severity classification when using GOLD 2011; 2) moderate agreement between mMRC and CAT scales suggests that the type of questionnaire used to evaluate perception of dyspnea can affect disease severity categorization; 3) group B patients showed a significant gas exchange impairment due to lower values of DLCO and arterial oxymetry and 4) a significant proportion of patients were categorized in the high-risk groups (B and D) both in GOLD 2011 and 2019. Optimization of the evaluation of COPD severity is important to allow a better standardization of care and pharmacological management of patients with this disease.

Key words: Pulmonary Disease, Chronic Obstructive, Spirometry, Forced Expiratory Volume, Observational study, Dyspnea.

\section{Resumen}

Introducción: La Enfermedad Pulmonar Obstructiva Crónica afecta a 260 millones de personas a nivel mundial y representará la tercera causa de muerte para el año 2020. Materiales y Métodos: Se realizó un estudio observacional descriptivo transversal con la finalidad de comparar la estadificación de un grupo de pacientes venezolanos con EPOC según la Global Initiative for Chronic Obstructive Lung Disease (GOLD) 2007, 2011 y 2019. Resultados: La muestra estuvo constituida por ochenta y nueve (89) pacientes con una edad promedio de 66,7 $\pm 0,9$ años, siendo el 60,7\% de los pacientes del sexo masculino y $82 \%$ fumadores. El 14,6\% de los pacientes presentaban EPOC leve, 36\% EPOC moderado, 41,6\% EPOC severo y 7,9\% EPOC muy severo. El valor del test Kappa de Cohen entre las escalas mMRC y CAT (COPD Assessment Test) fue de 0,529 (GOLD 2011) y 0,555 (GOLD 2019). Conclusiones: 1) la poca concordancia entre el VEF , grado de disnea e historial de exacerbaciones impacta la clasificación de la severidad de la EPOC al utilizar GOLD 2011;2) la concordancia moderada entre las escalas $\mathrm{mMRC}$ y CAT sugiere que el tipo de cuestionario utilizado afecta la categorización de la severidad de la enfermedad; 3) los pacientes del grupo B mostraron una importante afectación en el intercambio gaseoso dado por valores más bajos de DLCO y oximetría arterial y 4) una proporción significativa de pacientes fueron clasificados en los grupos de alto riesgo (B y $D)$ en GOLD 2011 y 2019.

Palabras clave: Enfermedad pulmonar obstructiva crónica, Espirometría, Volumen Espiratorio Forzado, Estudio Observacional, Disnea.

* Departamento de Ciencias Funcionales, Decanato de Ciencias de la Salud, Universidad Centroccidental "Lisandro Alvarado" (UCLA), Barquisimeto, Venezuela. 


\section{Introducción}

Los pacientes afectados por la Enfermedad Pulmonar Obstructiva Crónica (EPOC) tienen una elevada morbilidad y por ende un deterioro significativo en su calidad de vida asociado a un gran riesgo de muerte a pesar de los avances terapéuticos actuales. Esta enfermedad ocupará la tercera causa mundial de muerte para el año 2020 por lo que en la actualidad representa un problema de salud pública de primer orden con elevados costos socio-sanitarios tanto para los pacientes y familiares así como para los sistemas nacionales e internacionales de salud.

La Global Initiative for Chronic Obstructive Lung Disease (GOLD) es un consenso internacional auspiciado por el National Heart, Lung and Blood Institute (NHLBI) y la Organización Mundial de la Salud (OMS) cuya finalidad es unificar criterios en cuanto al diagnóstico, estadificación y seguimiento de los pacientes con EPOC. A partir del año 2007, la iniciativa GOLD clasificó la severidad de la EPOC en base al grado de limitación del flujo aéreo en los pacientes con una relación $\mathrm{VEF}_{1} / \mathrm{CVF}$ postbroncodilator $<0,70$ obteniéndose de esta forma cuatro estadios: GOLD I o leve $\left(\mathrm{VEF}_{1} \geq 80 \% \mathrm{del}\right.$ valor predictivo), GOLD II o moderado ( $50 \%$ $\leq \mathrm{VEF}_{1}<80 \%$ del valor predictivo), GOLD III o severo $\left(30 \% \leq \mathrm{VEF}_{1}<50 \%\right.$ del valor predictivo) y GOLD IV o muy severo ( $\mathrm{VEF}_{1}<30 \%$ del valor predictivo) ${ }^{1}$. A partir del año 2011 GOLD propuso una clasificación combinada la cual incorpora, además de la función pulmonar, variables clínicas tales como severidad de los síntomas (en base a los resultados obtenidos de la aplicación del cuestionario del Medical Research Council o del test de calidad de vida en la EPOC "CAT") e historial de exacerbaciones. Como resultado de esta nueva clasificación, el paciente puede categorizarse en cuatro grupos: A (bajo riesgo, poco sintomático), B (bajo riesgo, más sintomático), $\mathrm{C}$ (alto riesgo, poco sintomático) o $\mathrm{D}$ (alto riesgo, más sintomático) ${ }^{2}$. Desde el año 2017 y hasta la presente fecha, la GOLD propone una evaluación de la severidad de la enfermedad de forma más individualizada determinando en primer lugar la severidad de la limitación del flujo aéreo del paciente seguida de la cuantificación de sus síntomas y riesgo de exacerbaciones ${ }^{3}$.

El presente estudio fue realizado con la finalidad de comparar la estadificación de la severidad de la EPOC en un grupo de pacientes de acuerdo a GOLD 2007, 2011 y 2019.

\section{Materiales y Métodos}

Se realizó un estudio observacional descriptivo transversal donde se incluyeron 89 pacientes portadores de EPOC referidos de la consulta de Neumología del Hospital Universitario "Dr. Luis Gómez López", Hospital del Seguro Social "Dr. Pastor Oropeza" y de consultas privadas neumológicas de la ciudad de Barquisimeto, estado Lara, Venezuela en el lapso 2012-2015. Los criterios de inclusión fueron edad $\geq 40$ años, antecedentes de tabaquismo o exposición al humo de leña y espirometría con una relación $\mathrm{VEF}_{1} /$ CVF postbroncodilatador $<0,70$. Los criterios de exclusión fueron exacerbaciones (infecciosas y no infecciosas) en el mes previo o en el momento de ingreso al estudio, cáncer pulmonar, deterioro grave del sistema músculo-esquelético, infarto del miocardio en los últimos 6 meses y tromboembolismo pulmonar. El proyecto fue aprobado por la Comisión de Bioética del Decanato de Ciencias de la Salud de la Universidad Centroccidental "Lisandro Alvarado" (UCLA). Se obtuvo el consentimiento informado de cada paciente y todos los procedimientos médicos fueron realizados en concordancia con los principios éticos establecidos en la Declaración de Helsinki.

Una vez seleccionados los pacientes, se procedió a realizar sus respectivas historias clínicas $\mathrm{y}$ pruebas de función pulmonar utilizando un pletismógrafo Medgraphics Modelo Elite Dx 830001-008 según los criterios y recomendaciones de la ATS/ERS ${ }^{4-6}$. A todos los pacientes se les realizó la prueba de la caminata de los seis minutos y el cálculo del índice BODE como se ha descrito anteriormente ${ }^{7}$. Se evaluó la severidad de la disnea a través de la escala del Medical Research Council (mMRC) ${ }^{8}$ y del test de calidad de vida en la EPOC "CAT" y de esta forma se determinó si el paciente era menos sintomático (puntaje mMRC 0 -1 o puntaje CAT < 10) o más sintomático (puntaje mMRC $\geq 2$ o puntaje CAT $\geq 10$ ). Las exacerbaciones fueron definidas como episodios de aumento de síntomas (tos o disnea) que se acompañaron de uso de antibióticos y/o esteroides o la admisión a un centro de salud. Los pacientes de bajo riesgo fueron aquellos con $\leq 1$ exacerbaciones por año y ninguna hospitalización por exacerbaciones mientras que los pacientes de alto riesgo fueron aquellos con $\geq 2$ exacerbaciones por año $o \geq 1$ con hospitalización. En base a estas variables, se clasificó a los pacientes en los grupos $\mathrm{A}, \mathrm{B}, \mathrm{C}$ y $\mathrm{D}^{2,3}$.

El análisis de los datos se llevó a cabo utilizando el paquete estadístico SPSS versión 20 para Windows. Las variables cuantitativas se 
presentan como frecuencia absoluta, porcentajes o media \pm ES. La comparación de variables continuas se realizó con el test de Kruskal-Wallis. La comparación de variables categóricas se realizó con la prueba del $\chi^{2}$. Se utilizó el test Kappa de Cohen ${ }^{10}$ para evaluar la concordancia entre las clasificaciones según las diferentes versiones de GOLD. La significancia estadística se estableció para un valor de $\mathrm{p} \leq 0,05$.

\section{Resultados}

Las características clínicas y funcionales de los pacientes evaluados en este estudio de acuerdo a la clasificación GOLD 2007, 2011 y 2019 se muestran en las Tablas 1, 2 y 3. En general, la edad promedio de los pacientes fue de $66,7 \pm 0,9$ años, siendo el $60,7 \%$ de los pacientes del sexo masculino y $82 \%$ fumadores. Según la limitación del flujo aéreo (GOLD 2007), el 14,6\% de los pacientes fueron clasificados con EPOC I o leve, $36 \%$ con EPOC II o moderado, $41,6 \%$ con EPOC III o severo y $7,9 \%$ con EPOC IV o muy severo.

La clasificación GOLD 2011 incorpora, además de la función pulmonar, la valoración clínica del paciente en base a la cuantificación de la disnea y al número de exacerbaciones. Para cuantificar la severidad de la disnea y el impacto de la enfermedad en la calidad de vida de los pacientes con EPOC se utilizó la escala modificada del Medical Research Council británico (mMRC)

Tabla 1. Características de los pacientes participantes según GOLD 2007

\begin{tabular}{|c|c|c|c|c|c|}
\hline Variables & Estadio I & Estadio II & Estadio III & Estadio IV & p \\
\hline Edad (años) & $70,8 \pm 3$ & $67,1 \pm 1,5$ & $65,6 \pm 1,3$ & $63,3 \pm 3,5$ & 0,42 \\
\hline $\operatorname{Sexo}(M / F)(\%)$ & $38 / 62$ & $39 / 61$ & $42 / 58$ & $37 / 63$ & 0,94 \\
\hline Tabaquismo (\%) & 84 & 72 & 86 & 100 & 0,22 \\
\hline CT (paq/año) & $56 \pm 17$ & $36 \pm 8$ & $42 \pm 6$ & $65 \pm 20$ & 0,34 \\
\hline IMC $\left(\mathrm{kg} / \mathrm{m}^{2}\right)$ & $22,4 \pm 0,6$ & $26,4 \pm 0,9$ & $25,2 \pm 0,8$ & $22,1 \pm 1,9$ & 0,94 \\
\hline VEF post BD (L) & $2,1 \pm 0,1$ & $1,5 \pm 0,4$ & $1 \pm 0,7$ & $0,6 \pm 0,1$ & 0,04 \\
\hline VEF (\% pred) & $89 \pm 8$ & $62 \pm 1,2$ & $41 \pm 0,8$ & $25 \pm 2,2$ & 0,06 \\
\hline VEF/CVF (\%) & $62 \pm 1,7$ & $59 \pm 1,1$ & $51 \pm 1,3$ & $42 \pm 1,8$ & 0,44 \\
\hline $\mathrm{FEF}_{25-75 \%}(\mathrm{~L} / \mathrm{s})$ & $1,3 \pm 0,1$ & $0,9 \pm 0,0$ & $0,5 \pm 0,0$ & $0,3 \pm 0,1$ & 0,17 \\
\hline FEF (\% pred) & $70 \pm 6$ & $45 \pm 2,6$ & $25 \pm 1,5$ & $14 \pm 1,6$ & 0,21 \\
\hline CPT (L) & $5,8 \pm 0,3$ & $5,6 \pm 0,2$ & $6 \pm 0,2$ & $5,8 \pm 0,4$ & 0,76 \\
\hline CPT (\% pred) & $107 \pm 3,5$ & $107 \pm 3,3$ & $114 \pm 4,2$ & $112 \pm 9$ & 0,96 \\
\hline $\mathrm{CI}(\mathrm{L})$ & $2,1 \pm 0,2$ & $1,8 \pm 0,1$ & $1,6 \pm 0,1$ & $1,4 \pm 0,3$ & 0,57 \\
\hline CI (\% pred) & $85 \pm 6,2$ & $74 \pm 5,9$ & $69 \pm 4$ & $53 \pm 7,9$ & 0,72 \\
\hline VR (L) & $2,5 \pm 0,1$ & $2,8 \pm 0,2$ & $3,5 \pm 0,1$ & $3,8 \pm 0,4$ & 0,93 \\
\hline VR (\% pred) & $121 \pm 7,4$ & $143 \pm 57$ & $178 \pm 57$ & $211 \pm 24$ & 0,64 \\
\hline $\mathrm{RVA}(\mathrm{kPa} / \mathrm{L} / \mathrm{s})$ & $1 \pm 0,3$ & $0,8 \pm 0,2$ & $2,1 \pm 0,3$ & $3,2 \pm 1,2$ & 0,26 \\
\hline RVA (\% pred) & $225 \pm 47$ & $362 \pm 28$ & $534 \pm 70$ & $877 \pm 245$ & 0,20 \\
\hline DLCO & $8,9 \pm 1,1$ & $7 \pm 0,6$ & $3,6 \pm 2,6$ & $4,6 \pm 1,2$ & 0,24 \\
\hline DLCO (\% pred) & $78 \pm 6,9$ & $81 \pm 5,7$ & $39 \pm 13$ & $37 \pm 4$ & 0,05 \\
\hline 6MWT (m) & $437 \pm 33$ & $354 \pm 19$ & $351 \pm 16$ & $341 \pm 38$ & 0,43 \\
\hline $\mathrm{SAT}_{2}(\%)$ & $95 \pm 0,6$ & $94 \pm 0,3$ & $91 \pm 1$ & $89 \pm 1,5$ & 0,26 \\
\hline FC (lpm) & $73 \pm 3,5$ & $82 \pm 3$ & $81 \pm 2$ & $88 \pm 4$ & 0,06 \\
\hline PAS (mmHg) & $126 \pm 4,0$ & $129 \pm 2,7$ & $132 \pm 3,7$ & $124 \pm 11$ & 0,45 \\
\hline PAD (mmHg) & $69 \pm 2,5$ & $76 \pm 2,1$ & $77 \pm 2,1$ & $75 \pm 3$ & 0,01 \\
\hline
\end{tabular}

Los valores se presentan como frecuencia absoluta, porcentaje o media \pm ES. CT: carga tabáquica; IMC: índice de masa corporal; VEF post BD: volumen espiratorio forzado en el primer segundo postbroncodilatador; CVF: capacidad vital forzada; $\mathrm{FEF}_{25-75 \%}$ : flujo espiratorio forzado 25-75\%; CPT: capacidad pulmonar total; CI: capacidad inspiratoria; VR: volumen residual; RVA: resistencia de la vía aérea; DLCO: capacidad de difusión de monóxido de carbono expresado en $\mathrm{mM} / \mathrm{min} / \mathrm{kPa}$; 6MWT: caminata de $6 \mathrm{~min} ; \mathrm{SATO}_{2}$ : saturación arterial de oxígeno; FC: frecuencia cardíaca; PAS: presión arterial sistólica acostado; PAD: presión arterial diastólica acostado. 
Tabla 2. Características de los pacientes participantes según GOLD 2011

\begin{tabular}{|lccccc|}
\hline Variables & GOLD A & GOLD B & GOLD C & GOLD D & p \\
\hline Edad (años) & $62,2 \pm 5,3$ & $65,8 \pm 2$ & $70,7 \pm 3,4$ & $69,3 \pm 2,9$ & 0,34 \\
\hline Sexo (M/F) (\%) & $80 / 20$ & $55 / 45$ & $55 / 45$ & $36 / 64$ & 0,43 \\
\hline Tabaquismo (\%) & 80 & 83 & 77 & 54 & 0,36 \\
\hline CT (paq/año) & $39 \pm 20$ & $61 \pm 14$ & $22 \pm 8,3$ & $25 \pm 13$ & 0,33 \\
\hline IMC (kg/m²) & $23,7 \pm 2,4$ & $26,1 \pm 1,3$ & $24,3 \pm 1,5$ & $26,8 \pm 1,4$ & 0,54 \\
\hline VEF post BD (L) & $1,1 \pm 0,2$ & $0,9 \pm 0,0$ & $1,8 \pm 0,2$ & $1,3 \pm 0,1$ & 0,00 \\
\hline VEF (\% pred) & $37 \pm 4,0$ & $37 \pm 7,1$ & $78 \pm 4,2$ & $66 \pm 2,5$ & 0,00 \\
\hline VEF/CVF (\%) & $59 \pm 1,7$ & $60 \pm 1,2$ & $49 \pm 2,0$ & $50 \pm 1,5$ & 0,00 \\
\hline FEF $25-75 \%$ (L/s) & $0,6 \pm 0,1$ & $0,4 \pm 0,0$ & $1,1 \pm 0,1$ & $0,7 \pm 0,0$ & 0,00 \\
\hline FEF (\% pred) & $22 \pm 2,7$ & $23 \pm 3$ & $59 \pm 4,8$ & $49 \pm 6,6$ & 0,00 \\
\hline CPT (L) & $6,2 \pm 0,2$ & $6,1 \pm 0,3$ & $6,1 \pm 0,4$ & $4,8 \pm 0,3$ & 0,09 \\
\hline CPT (\% pred) & $109 \pm 13,7$ & $117 \pm 7,4$ & $113 \pm 3,8$ & $103 \pm 6,9$ & 0,75 \\
\hline CI (L) & $2,6 \pm 0,4$ & $1,4 \pm 0,0$ & $2,2 \pm 0,3$ & $1,3 \pm 0,2$ & 0,05 \\
CI (\% pred) & $83 \pm 7,4$ & $60 \pm 4,8$ & $92 \pm 10,3$ & $65 \pm 11$ & 0,03 \\
\hline VR (L) & $3,2 \pm 0,5$ & $3,9 \pm 0,2$ & $2,6 \pm 0,1$ & $2,6 \pm 0,4$ & 0,04 \\
\hline VR (\% pred) & $181 \pm 25$ & $194 \pm 15$ & $129 \pm 11$ & $130 \pm 24$ & 0,03 \\
\hline RVA (kPa/L/s) & $2 \pm 1,2$ & $2,6 \pm 0,6$ & $0,6 \pm 0,1$ & $1,7 \pm 0,8$ & 0,03 \\
\hline RVA (\% pred) & $629 \pm 242$ & $541 \pm 66$ & $318 \pm 57$ & $370 \pm 46$ & 0,09 \\
\hline DLCO & $6,6 \pm 2$ & $0,5 \pm 5,1$ & $9,6 \pm 1,6$ & $8,5 \pm 1,4$ & 0,05 \\
\hline DLCO (\% pred) & $59 \pm 25$ & $18 \pm 24$ & $95 \pm 16$ & $92 \pm 4,5$ & 0,00 \\
\hline 6MWT (m) & $425 \pm 6$ & $318 \pm 22$ & $430 \pm 32$ & $305 \pm 39$ & 0,01 \\
\hline SAT0 $(\%)$ & $90 \pm 2$ & $89 \pm 2$ & $95 \pm 0,7$ & $93 \pm 0,5$ & 0,03 \\
\hline FC (lpm) & $80 \pm 4,8$ & $82 \pm 3,2$ & $84 \pm 3,7$ & $80 \pm 7,4$ & 0,85 \\
\hline PAS (mmHg) & $135 \pm 9,8$ & $135 \pm 6,3$ & $128 \pm 6,6$ & $132 \pm 3,8$ & 0,93 \\
\hline PAD (mmHg) & $79 \pm 6,4$ & $80 \pm 2,9$ & $73 \pm 3,4$ & $76 \pm 3,2$ & 0,48 \\
\hline Los vas & & & & \\
\hline
\end{tabular}

Los valores se presentan como frecuencia absoluta, porcentaje o media \pm ES. CT: carga tabáquica; IMC: índice de masa corporal; VEF post BD: volumen espiratorio forzado en el primer segundo postbroncodilatador; CVF: capacidad vital forzada; $\mathrm{FEF}_{25-75 \%}$ : flujo espiratorio forzado $25-75 \%$; $\mathrm{CPT}$ : capacidad pulmonar total; CI: capacidad inspiratoria; VR: volumen residual; RVA: resistencia de la vía aérea; DLCO: capacidad de difusión de monóxido de carbono expresado en $\mathrm{mM} / \mathrm{min} / \mathrm{kPa} ; 6 \mathrm{MWT}$ : caminata de $6 \mathrm{~min} ; \mathrm{SATO}_{2}$ : saturación arterial de oxígeno; $\mathrm{FC}$ : frecuencia cardíaca; PAS: presión arterial sistólica acostado; PAD: presión arterial diastólica acostado.

o el COPD Assessment Test (CAT). La categorización de los pacientes en base a la clasificación GOLD 2011 (Tabla 4) utilizando la escala mMRC mostró que el $51,6 \%$ de los pacientes no pudieron ser clasificados debido a la discordancia entre los valores del $\mathrm{VEF}_{1}$, severidad de la disnea e historial de exacerbaciones. El 48,4\% de los pacientes restantes pudieron ser clasificados de la siguiente forma: 5,6\% GOLD A, 20,2\% GOLD B, 10,1\% GOLD C y $12,3 \%$ GOLD D. Asimismo, el 53,9\% de los pacientes no pudieron ser categorizados según el GOLD 2011 al utilizar la escala CAT. El resto de los pacientes se clasificaron de la siguiente manera: 1,1\% GOLD A, 23,5\% GOLD B y $21,3 \%$ como GOLD D. No hubo pacientes en el grupo C. El valor del test de concordancia Kappa de Cohen entre el mMRC y el CAT fue de $0,529(\mathrm{p}<0,0001)$.

La clasificación GOLD 2019 separa la categorización funcional (limitación del flujo aéreo) de la clínica (grado de disnea e historial de exacerbaciones). La clasificación de los pacientes en base a GOLD 2019 (Tabla 5) utilizando el mMRC reveló que $29,2 \%$ de los pacientes pertenecían a GOLD A, 25,8\% a GOLD B, 17,9\% a GOLD C y $26,9 \%$ a GOLD D. La categorización 
Tabla 3. Características de los pacientes participantes según GOLD 2019

\begin{tabular}{|c|c|c|c|c|c|}
\hline Variables & GOLD A & GOLD B & GOLD C & GOLD D & p \\
\hline Edad (años) & $63,9 \pm 1,7$ & $68,2 \pm 1,9$ & $67,7 \pm 2,4$ & $67,7 \pm 1,8$ & 0,50 \\
\hline $\operatorname{Sexo}(M / F)(\%)$ & $80 / 20$ & $52 / 48$ & $56 / 44$ & $50 / 50$ & 0,09 \\
\hline Tabaquismo (\%) & 88 & 78 & 87 & 75 & 0,55 \\
\hline CT (paq/año) & $49 \pm 8,2$ & $57 \pm 13$ & $21 \pm 4,6$ & $37 \pm 6,7$ & 0,16 \\
\hline $\operatorname{IMC}\left(\mathrm{kg} / \mathrm{m}^{2}\right)$ & $24,7 \pm 0,9$ & $25,9 \pm 1,1$ & $23,3 \pm 1,0$ & $25,3 \pm 1,0$ & 0,39 \\
\hline VEF post BD (L) & $1,7 \pm 0,1$ & $1,0 \pm 0,0$ & $1,5 \pm 0,1$ & $1,1 \pm 0,0$ & 0,00 \\
\hline VEF (\% pred) & $62 \pm 3,8$ & $44 \pm 3,6$ & $62 \pm 5,1$ & $49 \pm 3,5$ & 0,00 \\
\hline VEF/CVF (\%) & $59 \pm 1,7$ & $60 \pm 1,2$ & $49 \pm 2,0$ & $50 \pm 1,5$ & 0,10 \\
\hline $\mathrm{FEF}_{25-75 \%}(\mathrm{~L} / \mathrm{s})$ & $1,0 \pm 0,1$ & $0,5 \pm 0,0$ & $0,9 \pm 0,1$ & $0,5 \pm 0,0$ & 0,01 \\
\hline FEF (\% pred) & $41 \pm 3,5$ & $33 \pm 5,4$ & $43 \pm 5,5$ & $34 \pm 4,1$ & 0,05 \\
\hline CPT (L) & $6,1 \pm 0,2$ & $5,9 \pm 0,3$ & $5,5 \pm 0,3$ & $5,5 \pm 0,3$ & 0,65 \\
\hline CPT (\% pred) & $110 \pm 3,9$ & $115 \pm 7,1$ & $108 \pm 4,2$ & $111 \pm 4,7$ & 0,77 \\
\hline $\mathrm{CI}(\mathrm{L})$ & $2,2 \pm 0,1$ & $1,4 \pm 0,0$ & $1,8 \pm 0,2$ & $1,6 \pm 0,1$ & 0,03 \\
\hline CI (\% pred) & $81 \pm 5,1$ & $61 \pm 3,9$ & $78 \pm 8,4$ & $69 \pm 6,4$ & 0,00 \\
\hline VR (L) & $2,9 \pm 0,1$ & $3,6 \pm 0,2$ & $2,8 \pm 0,1$ & $3,2 \pm 0,2$ & 0,15 \\
\hline VR (\% pred) & $154 \pm 9,2$ & $178 \pm 14,1$ & $141 \pm 11$ & $163 \pm 15,8$ & 0,25 \\
\hline $\mathrm{RVA}(\mathrm{kPa} / \mathrm{L} / \mathrm{s})$ & $0,9 \pm 0,2$ & $2,2 \pm 0,5$ & $1,5 \pm 0,6$ & $1,8 \pm 0,4$ & 0,01 \\
\hline RVA (\% pred) & $361 \pm 62$ & $495 \pm 55$ & $448 \pm 103$ & $542 \pm 110$ & 0,12 \\
\hline DLCO & $7,0 \pm 0,5$ & $1,3 \pm 3,9$ & $7,7 \pm 1,2$ & $7,5 \pm 0,9$ & 0,02 \\
\hline DLCO (\% pred) & $71 \pm 6,0$ & $28 \pm 19$ & $77 \pm 12$ & $72 \pm 5,1$ & 0,00 \\
\hline 6MWT (m) & $412 \pm 16$ & $313 \pm 20$ & $414 \pm 21$ & $318 \pm 25$ & 0,00 \\
\hline $\mathrm{SATO}_{2}(\%)$ & $94 \pm 0,6$ & $90 \pm 1,5$ & $94 \pm 0,6$ & $91 \pm 0,6$ & 0,00 \\
\hline $\mathrm{FC}(\mathrm{lpm})$ & $74 \pm 3,0$ & $82 \pm 2,6$ & $82 \pm 3,1$ & $83 \pm 3,6$ & 0,07 \\
\hline PAS (mmHg) & $127 \pm 3,2$ & $135 \pm 5,2$ & $124 \pm 5,1$ & $130 \pm 4,0$ & 0,48 \\
\hline PAD (mmHg) & $75 \pm 2,8$ & $79 \pm 2,6$ & $71 \pm 2,7$ & $76 \pm 2,0$ & 0,37 \\
\hline
\end{tabular}

Los valores se presentan como frecuencia absoluta, porcentaje o media \pm ES. CT: carga tabáquica; IMC: índice de masa corporal; VEF post BD: volumen espiratorio forzado en el primer segundo postbroncodilatador; CVF: capacidad vital forzada; $\mathrm{FEF}_{25-75 \%}$ : flujo espiratorio forzado 25-75\%; CPT: capacidad pulmonar total; CI: capacidad inspiratoria; VR: volumen residual; RVA: resistencia de la vía aérea; DLCO: capacidad de difusión de monóxido de carbono expresado en $\mathrm{mM} / \mathrm{min} / \mathrm{kPa}$; 6MWT: caminata de $6 \mathrm{~min} ; \mathrm{SATO}_{2}$ : saturación arterial de oxígeno; FC: frecuencia cardíaca; PAS: presión arterial sistólica acostado; PAD: presión arterial diastólica acostado.

Tabla 4. Tabla de clasificación cruzada mostrando la distribución de los pacientes por categorías según GOLD 2011

\begin{tabular}{|lccccrr|r|}
\hline & & \multicolumn{2}{c}{ Estadio GOLD 2011 (CAT) } & \multicolumn{2}{c|}{ Total } \\
Estadio GOLD 2011 (mMRC) & & A & B & C & D & 5 \\
& A & 1 & 4 & 0 & 0 & 16 \\
& B & 0 & 16 & 0 & 0 & 9 \\
Total & C & 0 & 1 & 0 & 8 & 11 \\
& D & 0 & 0 & 0 & 11 & 41 \\
\hline
\end{tabular}

mMRC $=$ escala modificada de disnea del Medical Research Council británico; CAT = COPD Assessment test (test de calidad de vida en pacientes con EPOC). 
Tabla 5. Tabla de clasificación cruzada mostrando la distribución de los pacientes por categorías según GOLD 2019

\begin{tabular}{|lcccccc|}
\hline & & \multicolumn{4}{c}{ Estadio GOLD 2019 (CAT) } & Total \\
Estadio GOLD 2019 (mMRC) & A & 12 & 14 & 0 & 0 & 26 \\
& B & 0 & 23 & 0 & 0 & 23 \\
& C & 0 & 0 & 1 & 15 & 16 \\
Total & D & 0 & 0 & 0 & 24 & 24 \\
\hline
\end{tabular}

mMRC = escala modificada de disnea del Medical Research Council británico; CAT = COPD Assessment test (test de calidad de vida en pacientes con EPOC).

de los pacientes utilizando el CAT reveló que $13,4 \%$ de los pacientes fueron clasificados como GOLD A, 40,4\% como GOLD B, 1,12\% como GOLD C y $43,8 \%$ como GOLD D. La totalidad de los pacientes previamente clasificados en los grupos A, B y C de acuerdo a GOLD 2011 permanecieron en sus mismos grupos en la clasificación GOLD 2019 excepto para los pacientes pertenecientes al grupo D. El 75\% (mMRC) y el $83 \%$ (CAT) de los pacientes del grupo D permanecieron en su mismo grupo y el resto fue reclasificado al grupo B. Los pacientes del grupo $B$ presentaron valores similares de capacidad inspiratoria $(\mathrm{CI})$ y de distancia caminada en $6 \mathrm{~min}$ (6MWT) en comparación con el grupo D, sin embargo, mostraron valores comparativamente más bajos de capacidad de difusión de monóxido de carbono (DLCO) y de saturación arterial de oxígeno $\left(\mathrm{SATO}_{2}\right)$ así como valores significativamente más elevados de volumen residual (VR). El valor del test de concordancia Kappa de Cohen entre el mMRC versus CAT (GOLD 2019) fue de $0,555(\mathrm{p}<0,0001)$. El valor del test de concordancia kappa de Cohen entre el GOLD $2007 \mathrm{y}$ GOLD 2019 fue de $-0,009$ (mMRC, $p=0,871)$ y $-0,008$ (CAT, $\mathrm{p}=0,863$ ), respectivamente.

\section{Discusión}

La utilidad de la clasificación de la severidad de la EPOC para predecir el riesgo de muerte según la Iniciativa Global para la Enfermedad Pulmonar Obstructiva Crónica (GOLD) ha sido previamente establecida ${ }^{11,12}$. Tradicionalmente, la categorización de la severidad de la enfermedad se ha realizado en base al grado de afectación del $\mathrm{VEF}_{1}$; sin embargo, diversos estudios apuntan a que la medición de la limitación al flujo aéreo no refleja de forma fehaciente el impacto que tiene la EPOC en la calidad de vida de los pacientes.
Por esta razón, GOLD ha propuesto un abordaje multidimensional incorporando en la estadificación de la enfermedad variables clínicas tales como severidad de los síntomas e historial de exacerbaciones. El objetivo de este estudio fue el de comparar la clasificación tradicional de la severidad de la EPOC versus versiones más recientes de esta clasificación en un grupo de pacientes venezolanos.

De acuerdo a los criterios de clasificación GOLD 2007, la mayor parte de los pacientes de este estudio presentaron EPOC moderado a severo $(\sim 78 \%)$. Al utilizar los criterios GOLD 2011, más de la mitad de los pacientes no pudieron ser reclasificados. Estos resultados apuntan a la pobre correlación que existe entre la limitación crónica al flujo aéreo y variables clínicas tales como grado de percepción de la disnea, exacerbaciones, etc. ${ }^{13-17}$. El uso de los criterios GOLD 2019 demostró cambios significativos en la distribución de los pacientes y permitió que todos éstos pudieran ser reclasificados en diferentes grupos observándose un mayor porcentaje de pacientes en el grupo A en comparación con la clasificación GOLD 2011. Al utilizar el cuestionario CAT se pudo observar una distribución de los pacientes hacia los grupos más sintomáticos y de mayor riesgo (B y D), lo cual concuerda con publicaciones realizadas por otros autores ${ }^{18,19}$. En apoyo a nuestros hallazgos, un reciente estudio ha demostrado que los pacientes latinoamericanos con EPOC tienden a presentar una alta frecuencia de síntomas respiratorios ${ }^{20}$. Por otra parte, es importante mencionar que el grupo de pacientes de este estudio han sido recién diagnosticados con EPOC y que lamentablemente presentan la enfermedad en una fase avanzada por lo cual no es sorprendente que sean muy sintomáticos.

En nuestro estudio, el grupo C sigue representando la categoría con menor número de pacientes lo cual concuerda con los resultados obtenidos 
de los estudios de Copenhagen, COPDGene, entre otros ${ }^{21-23}$. Los pacientes categorizados en el grupo B mostraron un importante compromiso en su función pulmonar concretamente en variables fisiológicas asociadas al intercambio gaseoso. Estos resultados concuerdan con Ohgiya ${ }^{24}$ quienes demostraron una mayor prevalencia de anormalidades intersticiales pulmonares en este subgrupo lo cual en cierta forma explica una mayor tasa de mortalidad en estos pacientes ${ }^{22}$.

La discordancia entre la evaluación del grado de percepción de la disnea a través de la escala mMRC y CAT ha sido previamente descrita. Por ejemplo, Kim y cols..$^{25}$ demostraron que la clasificación de un grupo de pacientes con EPOC utilizando la escala mMRC o el cuestionario CAT no fue idéntica. Rieger-Reyes y cols. ${ }^{26}$ y Huang y cols. ${ }^{27}$ observaron que el $25 \%$ y $38,2 \%$ de los pacientes de sus respectivos estudios fueron reclasificados en diferentes grupos al utilizar estos cuestionarios. Por ejemplo, Rhee y cols. ${ }^{28}$ en su grupo de estudio evidenció que el número de pacientes en los grupos más sintomáticos según el cuestionario CAT disminuyó sustancialmente al clasificarlos con la escala mMRC. Al ser la escala mMRC una herramienta de evaluación de la disnea de esfuerzo y el CAT un cuestionario de calidad de vida relacionado con la salud no es sorprendente observar estas diferencias. En consonancia con esta observación, Chung y cols. ${ }^{29}$ demostraron que la escala mMRC se relaciona mejor con la capacidad para el ejercicio que con la percepción de la disnea de esfuerzo, mientras que el cuestionario CAT se correlaciona pobremente con la prueba de la caminata de $6 \mathrm{~min}$. Estos hallazgos sugieren que aun cuando el uso de ambos tipos de cuestionarios pudiera lograr una evaluación clínica más integral del paciente con EPOC, la categorización de la severidad de la enfermedad no es indiferente al uso de uno u otro método de evaluación de la percepción de la disnea.

Este estudio tiene una limitación en relación al tamaño muestral. A pesar de este inconveniente, nuestros resultados concuerdan con las publicaciones emanadas de otros centros de investigación, tanto a nivel primario como especializado. Una importante ventaja es el hecho de que los pacientes al momento del ingreso al estudio estaban siendo diagnosticados con la enfermedad por primera vez $y$, por lo tanto, no se encontraban recibiendo medicación de forma regular. El seguimiento de estos pacientes a través de un estudio prospectivo pudiera aportar datos significativos para evaluar el pronóstico y avance de la severidad de la enfermedad en los diferentes grupos. En conclusión, nuestros resultados indican la necesidad de reevaluar continuamente la clasificación de la severidad en el paciente con EPOC con el fin de optimizar el abordaje clínico y el manejo terapéutico de los pacientes afectados por esta enfermedad.

\section{Financiamiento}

Este proyecto fue financiado por el Consejo de Desarrollo Científico, Humanístico y Tecnológico (CDCHT) de la Universidad Centroccidental "Lisandro Alvarado" (Barquisimeto, Estado Lara, Venezuela) (código ME-2012-9).

\section{Agradecimientos}

Nuestro agradecimiento a los pacientes que participaron en este estudio así como al personal médico y de enfermería del Servicio de Neumonología y Cirugía de Tórax del Hospital Universitario "Dr. Luis Gómez López", Hospital del Seguro Social "Dr. Pastor Oropeza" y consultas privadas de la ciudad de Barquisimeto, Estado Lara. Se agradece a la Licenciada Elodia Delgado por su apoyo en la tabulación de los datos.

\section{Bibliografía}

1.- RABE K, HURD S, ANZUETO A, BARNES P, BUIST S, CALVERLEY P, et al. Global strategy for the Diagnosis, Management, and Prevention of Chronic Obstructive Pulmonary Disease: GOLD executive summary. Am J Respir Crit Care Med 2007; 176: 532-55.

2.- VESTBO J, HURD S, AGUSTI A, JONES P, VOGELMEIER C, ANZUETO A, et al. Global Strategy for the Diagnosis, Management, and Prevention of Chronic Obstructive Pulmonary Disease: GOLD executive summary. Am J Respir Crit Care Med 2013; 187: 347-65.

3.- https://goldcopd.org/wp-content/uploads/2018/11/ GOLD-2019-POCKET-GUIDE-FINAL_WMS.pdf.

4.- MILLER M, HANKINSON J, BRUSASCO V, BURGOS F, CASABURI R, COATES A, et al. Standardization of spirometry. Eur Respir J 2005; 26: 319-38.

5.- WANGER J, CLAUSEN J, COATES A, PEDERSEN O, BRUSASCO V, BURGOS F, et al. Standardization of the measurement of lung volumes. Eur Respir J 2005; 26: 511-22.

6.- MACINTYRE N, CRAPO R, VIEGI G, JOHNSON D, VAN DER GRITEN C, BRUSASCO V, et al. Standardization of the single-breath determination of carbon monoxide uptake in the lung. Eur Respir J 2005; 26: 720-35.

7.- CELLI B, COTE C, MARIN J, CASANOVA C, MONTES DE OCA M, MÉNDEZ R, et al. The Body-Mass index, airflow obstruction, dyspnea and exercise capa- 
city index in chronic obstructive pulmonary disease. $\mathrm{N}$ Engl J Med 2004; 350: 1005-12.

8.- BESTALL J, PAUL E, GARROD R, GARNHAM R, JONES P, WEDZICHA J. Usefulness of the Medical Research Council (MRC) dyspnoea scale as a measure of disability in patients with chronic obstructive pulmonary disease. Thorax 1999; 54: 581-6.

9.- JONES P, HARDING G, BERRY P, WIKLUND I, CHEN W, KLINE L. Development and first validation of the COPD Assessment Test. Eur Respir J 2009; 34: 648-54.

10.- COHEN J. A coefficient of agreement for nominal scales. Educ Psychol Meas 1960; 20: 37-46.

11.- MANNINO D, DOHERTY D, SONIA BUIST A. Global Initiative on Obstructive Lung Disease (GOLD) classification of lung disease and mortality: findings from the Atherosclerosis Risk in Communities (ARIC) study. Respir Med 2006; 100: 115-22.

12.- PEDONE C, GIUA R, SCICHILONE N, BELLIA V, ANTONELLI-INCALZI R. GOLD staging system is appropriate to predict mortality in older people with Chronic Obstructive Pulmonary Disease. Arch Bronconeumol 2018; 100: 115-22.

13.- VAN DIJK W, TAN W, LI P, GUO B, LI S, BENEDETTI A, et al. Clinical relevance of fixed ratio vs lower limit of normal $\mathrm{FEV}_{1} / \mathrm{FVC}$ in COPD: Patient reported outcomes from the CanCOLD Cohort. Ann Fam Med 2015; $13: 41-8$.

14.- MONTES DE OCA M, LÓPEZ M, ACUÑAA, SCHIAVI E, REY M, JARDIN J, et al. ALAT-2014 Chronic Obstructive Pulmonar Disease (COPD) Clinical Practice Guidelines: Questions and Answers. Arch Bronconeumonol 2015; 51: 369-426.

15.- KIM J, LEE S, LEE B, LEE C, KIM D, MIN K, et al. Factors associated with exacerbation in mil-to-moderate COPD patients. Int J Chron Obstruct Pulmon Dis 2016; 11: 1327-33.

16.- AGUSTI A, CALVERLEY P, CELLI B, COXSON H, EDWARDS L, LOMAS D, et al. Characterization of COPD heterogeneity in the ECLIPSE cohort. Respir Res 2010; 11: 122.

17.- NISHIMURA K, IZUMI T, TSUKINO M, OGA T. Dyspnea is a better predictor of 5-year survival than airway obstruction in patients with COPD. Chest 2002; 121: 1434-40.

18.- HERNÁNDEZ M, GARCÍA G, FALCO J, GARCÍA A, MARTÍN V, IBARROLA M, et al. Impact of using the new GOLD classification on the distribution of COPD severity in clinical practice. Int J COPD 2018; 13: 3516.

19.- HAUGHNEY J, GRUFFYDD-JONES K, ROBERTS J, LEE A, HARDWELL A, MCGARVEY L. The distribution of COPD in UK general practice using the new GOLD classification. Eur Respir J 2014; 43: 993-1002.

20.- MIRATVILLES M, MENEZES A, LÓPEZ M, CASAS A, UGALDE L, RAMÍREZ-VENEGAS A, et al.
Prevalence and impact of respiratory symptoms in a population of patients with COPD in Latin America: The LASSYC Observational Study. Respir Med 2018; 134: 62-9.

21.- JOHANNESSEN A, NILSEN R, STOREBØ M, GULSVIK A, EAGAN T, BAKKE P. Comparison of 2011 and 2007 Global Initiative for Chronic Obstructive Lung Disease Guidelines for predicting Mortality and Hospitalization. Am J Respir Crit Care Med 2013; 188: 51-9.

22.- LANGE P, MAROTT J, VESTBO J, OLSEN K, INGEBRIGTSEN T, DAHL M, et al. Prediction of the clinical course of chronic obstructive pulmonary disease, using the new GOLD classification: a study of the general population. Am J Respir Crit Care Med 2012; 186: 975-81.

23.- HAN M, MUELLEROVA H, CURRAN-EVERETT D, DRANSFIELD M, WASHKO G, REGAN E et al. GOLD 2011 disease severity classification in COPDGene: a prospective cohort study. Lancet Respir Med 2013; 1: 43-50.

24.- OHGIYA M, MATSUI H, TAMURA A, KATO T, AKAGAWA S, OHTA K. The evaluation of interstitial abnormalities in group B of the 2011 Global Initiative for Chronic Obstructive Lung Disease (GOLD) classification of Chronic Obstructive Pulmonary Disease (COPD). Intern Med 2017; 56: 2711-17.

25.- KIM S, OH J, KIM Y, BAN H, KWON Y, OH I, et al. Differences in classification of COPD groups using COPD assessment test (CAT) or modified Medical Research Council (mMRC) dyspnea scores: a cross sectional analysis. BMC Pulm Med 2013; 13: 35.

26.- RIEGER-REYES C, GARCÍA-TIRADO F, RUBIOGALÁN F, MARIN-TRIGO J. Classification of chronic obstructive pulmonary disease severity according to the new Global Initiative for Chronic Obstructive Lung Disease 2011 guidelines: COPD assessment test versus modified Medical Research Council scale. Arch Bronconeumol 2014; 50: 129-34.

27.- HUANG W, WU M, CHEN H, HSU J, TOLD GROUP. Features of COPD patients by comparing CAT with mMRC: a retrospective, cross-sectional study. NPJ Prim Care Respir Med 2015; 25: 150-63.

28.- RHEE C, KIM J, HWANG Y, LEE J, JUNG K, LEE M, et al. Discrepancies between modified Medical Research Council dyspnea score and COPD assessment test score in patients with COPD. Int J Chron Obstruct Pulmon Dis 2015; 10: 1623-31.

29.- CHUNG M-L, LIN I-F, LEE C-Y. Clinical assessment tests in evaluating patients with chronic obstructive pulmonary disease. Medicine 2016; 95: 47(e5471).

Correspondencia a:

Dra. Joanna Santeliz C.

Departamento de Ciencias Funcionales,

Decanato de Ciencias de la Salud,

Universidad Centroccidental "Lisandro Alvarado"

(UCLA), Barquisimeto, Venezuela.

Email: jsanteliz@ucla.edu.ve 